\title{
Variation in local carrying capacity and the individual fate of bacterial colonizers in the phyllosphere
}

\author{
Mitja NP Remus-Emsermann ${ }^{1}$, Robin Tecon ${ }^{1,2}$, George A Kowalchuk ${ }^{1,3}$ and \\ Johan HJ Leveau ${ }^{1,2}$ \\ ${ }^{1}$ Department of Microbial Ecology, Netherlands Institute of Ecology (NIOO-KNAW), Wageningen, \\ The Netherlands; ${ }^{2}$ Department of Plant Pathology, University of California, Davis, CA, USA and \\ ${ }^{3}$ Department of Ecological Science, VU University Amsterdam, Amsterdam, The Netherlands
}

\begin{abstract}
Using a phyllosphere model system, we demonstrated that the term 'carrying capacity', as it is commonly used in microbial ecology, needs to be understood as the sum of many 'local carrying capacities' in order to better explain and predict the course and outcome of bacterial colonization of an environment. Using a green fluorescent protein-based bioreporter system for the quantification of reproductive success (RS) in individual Erwinia herbicola cells, we were able to reconstruct the contribution of individual immigrants to bacterial population sizes on leaves. Our analysis revealed that plant foliage represents to bacteria an environment where individual fate is determined by the local carrying capacity of the site where an immigrant cell lands. With increasing inoculation densities, the RS of most immigrants declined, suggesting that local carrying capacity under the tested conditions was linked to local nutrient availability. Fitting the observed experimental data to an adapted model of phyllosphere colonization indicated that there might exist three types of sites on leaves, which differ in their frequency of occurrence and local carrying capacity. Specifically, our data were consistent with a leaf environment that is characterized by few sites where individual immigrants can produce high numbers of offspring, whereas the remainder of the leaf offered an equal number of sites with low and medium RS. Our findings contribute to a bottom-up understanding of bacterial colonization of leaf surfaces, which includes a quantifiable role of chance in the experience at the individual level and in the outcome at the population level.

The ISME Journal (2012) 6, 756-765; doi:10.1038/ismej.2011.209; published online 19 January 2012

Subject Category: microbial population and community ecology

Keywords: individual-based ecology; environmental heterogeneity; bioreporter; phyllosphere; single-cell microbiology; modeling
\end{abstract}

\section{Introduction}

In ecology, the 'carrying capacity' of an environment is the maximum number of individuals that the environment can support (McArthur, 2006). In phyllosphere microbiology, that is, the study of microorganisms that colonize leaf surfaces, this term is invariably referred to as the number of microbial cells that a leaf is able to sustain (Wilson and Lindow, 1994a, b; Wilson et al., 1995; Woody et al., 2007; Nix et al., 2009). Carrying capacity of leaf surfaces is typically determined through counts of colony-forming units (CFUs) on agar plates. It is a conditional value that depends on several biotic and abiotic factors (Leveau, 2006). For example, different

Correspondence: JHJ Leveau, Department of Plant Pathology, University of California, One Shields Avenue, Davis, CA 95616, USA.

E-mail: jleveau@ucdavis.edu

Received 20 September 2011; revised 2 December 2011; accepted 3 December 2011; published online 19 January 2012 plant species are known to sustain different microbial population sizes (Kinkel et al., 2000; Knief et al., 2010b). Furthermore, bacterial species may vary in the densities which they can achieve on leaf surfaces depending on their ability to deal with the environmental conditions on the leaf surface (Jacques et al., 1995; Jacobs et al., 2005). Water availability on the leaf has frequently been highlighted as another driver for microbial abundance in the phyllosphere (Leben, 1965; Elad and Kirshner, 1993; Wilson and Lindow, 1994a, b).

One key factor in determining foliar carrying capacity is the availability of nutrients. Carbon is a limiting resource during phyllosphere colonization (Mercier and Lindow, 2000; Leveau and Lindow, 2001a,b). The process of leaching (Tukey, 1966) deposits photosynthates, such as the sugars fructose, sucrose and glucose, on the leaf surface where they become readily available for consumption by microbes (Mercier and Lindow, 2000; Leveau and Lindow, 2001a, b; van der Wal and Leveau, 2010). Spraying of a carbon source onto plant leaves 
(Wilson and Lindow, 1995) or making an additional carbon source available through genetic modification of the plant (Wilson et al., 1995) can also increase the carrying capacity of leaves for bacterial populations.

The use of bacterial bioreporters has confirmed that sugars, such as fructose, are not distributed evenly across the leaf surface (Leveau and Lindow, 2001a,b). It has also been reported that unused sugars can still be detected on leaves that are colonized by bacteria at the apparent carrying capacity (Mercier and Lindow, 2000). These two observations are in line with the hypothesized existence of discrete sites on the leaf surface that contain disparate amounts of resources (for example, sugars) and that may or may not be colonized by bacteria (Kinkel et al., 2002; Lindow and Brandl, 2003). At high inoculation densities, all sites on a leaf can be expected to be colonized by bacteria, and in these cases, carrying capacity will be determined by the amount of nutrients available in all sites combined. At low inoculation densities, some sites will remain uncolonized, including some that contain nutrients. In the latter case, the carrying capacity is not reached and instead becomes a function of how many, and which, sites are actually occupied by bacterial colonizers. This model of leaf colonization was partly verified in experiments with the epiphytic bacterium Pseudomonas syringae (Wilson and Lindow, 1994a, b), revealing that populations derived from low inoculum concentrations never were able to reach the carrying capacity that was realized at high inoculum densities. However, what is still lacking is an experimental, quantitative demonstration of the variation that exists in nutrient availability, or local carrying capacity, between sites on a leaf surface. How big is this variation, and how does it contribute to the course and outcome of leaf colonization? Population-based measurements are not suited to address these questions, as they only examine averages across the population as a whole. In order to tackle these issues properly, an individual-based approach seems necessary.

Here, we used the bacterial bioreporter CUSPER (reverse of REPSUC, for REProductive SUCcess; Remus-Emsermann and Leveau, 2010) to describe how increasing inoculation densities are experienced by individual bacterial colonizers of bean leaf surfaces. From this information, we were able to derive estimates for the variation in local carrying capacity and assess the impact of this variation on achieving final foliar population sizes. The CUSPER bioreporter is based on dilution of green fluorescent protein (GFP) from dividing cells in such a way that GFP fluorescence of a single cell is inversely proportional to the reproductive success (RS) of the original immigrant from which the observed cell was derived. The CUSPER bioreporter was recently used to reveal that in a heterogeneous environment, such as the phyllosphere, individual bacterial immigrants contribute unequally to future population sizes (Remus-Emsermann and Leveau, 2010). In this study, we utilized CUSPER for an individualbased examination of leaf colonization at different inoculation densities in order to gain a quantitative understanding of variation in local carrying capacities in the phyllosphere. Such knowledge is potentially important to general ecological understanding as well as practical applications such as plant pathogen control.

\section{Materials and methods}

Bacterial strain, culture conditions and plant experiments

CUSPER is a bacterial bioreporter of RS (RemusEmsermann and Leveau, 2010). It is based on Erwinia herbicola 299R::JBA28 (pCPP39; Leveau and Lindow, 2001a,b), which constitutively expresses LacI ${ }^{\mathrm{q}}$ from plasmid pCPP39 and carries a chromosomal mini-Tn5-Km insertion that expresses stable GFP from a LacI $^{\mathrm{q}}$-repressible $\mathrm{P}_{\mathrm{A} 1 / 04 / 03}$ promoter fusion to gfpmut3. The transposon confers resistance to kanamycin and the plasmid to tetracycline. The concept of CUSPER lies in the loading of bacterial cells with GFP through derepression of the $\mathrm{P}_{\mathrm{A} 1 / 04 / 03}$ promoter with isopropyl $\beta$-D-1thiogalactopyranoside, followed by release of these GFP-loaded cells into an $\beta$-D-1-thiogalactopyranoside-free environment. Without de novo GFP production, GFP is diluted every time a cell divides, so that GFP fluorescence in each cell becomes an inverse measure for RS. CUSPER cells were cultured to mid-exponential phase at 300 r.p.m. and $28^{\circ} \mathrm{C}$ in LB broth supplemented with $50 \mu \mathrm{g}$ kanamycin and $15 \mu \mathrm{g}$ tetracycline per $\mathrm{ml}$ in addition to $1 \mathrm{~mm} \beta$-D-1thiogalactopyranoside. Bacteria were harvested by centrifugation at $3900 \times g$ for $10 \mathrm{~min}$, and resuspended in $1 \times$ phosphate-buffered saline to approximate densities of $10^{5}, 10^{6}, 10^{7}$ and $10^{8} \mathrm{CFU} \mathrm{ml}^{-1}$. Two fully expanded cotyledon leaves of 14-day-old Phaseolus vulgaris plants (green snap bean, variety Blue Lake Bush 274; Burpee, Warminster, PA, USA) were inoculated by evenly spraying the adaxial surface of each leaf with $\sim 0.5 \mathrm{ml}$ of one of the four bacterial suspensions using an airbrush paint gun (Pilot I Spray gun, Walther Pilot, WuppertalVohwinkel, Germany). Inoculated plants were transferred to closed translucent boxes and incubated at high relative humidity and $21^{\circ} \mathrm{C}$.

Recovery of bacteria and analysis by microscopy After 0, 3, 5, 7 and $24 \mathrm{~h}$, three leaves were sampled from the plants that had been sprayed with one of the four bacterial suspensions, for a subtotal of 12 leaves per time point and a grand total of 60 leaves. Each leaf was transferred to a $50 \mathrm{ml}$ Falcon tube (BD Biosciences, Breda, The Netherlands) with $20 \mathrm{ml}$ $1 \times$ phosphate-buffered saline buffer, vortexed briefly, sonicated for $7 \mathrm{~min}$ and vortexed briefly 
again. Aliquots $(50 \mu \mathrm{l})$ from each leaf wash were plated using an Eddy Jet spiral plater (IUL, Barcelona, Spain) onto duplicate LB agar-plates supplemented with $50 \mu \mathrm{g}$ kanamycin per ml to determine CFUs.

For two of the three leaves per time point and treatment (that is, a total of 40 leaves), the remainder of the leaf wash was filtered over a $0.2-\mu \mathrm{m}$ Isopore filter (Millipore, Amsterdam, The Netherlands). CUSPER cells were recovered from the filter by vortexing for $15 \mathrm{~s}$ in $1 \mathrm{ml} 1 \times$ phosphate-buffered saline, fixed as previously described (Leveau and Lindow, 2001a,b) and stored in $1 \times$ phosphatebuffered saline at $4{ }^{\circ} \mathrm{C}$ for no longer than 3 days. Cells were analyzed with an Axio Imager M1 epifluorescent microscope (Zeiss, Oberkochen, Germany) using standard phase contrast and Zeiss Filter 38 (BP 470/ 40, FT 495, BP 525/ 50) for the visualization of GFP. Digital images were captured using an EC Plan-Neofluar $100 \times / 1.30$ oil objective (Zeiss) with an AxioCam MRm camera (Zeiss). Single-cell fluorescence was quantified as the mean pixel intensity of individual cells (Leveau and Lindow, 2001a, b) using the AxioVision 2.8 software package (Zeiss, Jena, Germany). Downstream data analysis was performed in Microsoft Excel 2010 (Microsoft Corporation, Redmond, WA, USA). To reconstruct the relative contribution of original leaf immigrants to the final population based upon single-cell green fluorescence measurements, we divided the green fluorescence of every one of the $N$ cells in each sample at $t=24$ by the average cell's green fluorescence in the inoculum at time $t=0$. For each cell, this relative GFP content was divided by the sum of all GFP contents within the same sample ( $\Sigma$ GFP) to give a GFP/ $\Sigma$ GFP value for each cell. For each cell, we also calculated the RS of its original progenitor as ${ }^{2} \log (1 / \mathrm{GFP})$. Cells were then binned into one of the following RS classes: $\mathrm{RS}<0.5,0.5 \leqslant \mathrm{RS}<1.5,1.5 \leqslant \mathrm{RS}<2.5,2.5 \leqslant \mathrm{RS}<3.5$, $3.5 \leqslant R S<4.5,4.5 \leqslant R S<5.5$ and $R S \geqslant 5.5$. For each bin, the GFP/ $\Sigma$ GFP values of all cells were summed to give $\Sigma(\mathrm{GFP} / \Sigma \mathrm{GFP})$. Values of $\Sigma(\mathrm{GFP} / \Sigma \mathrm{GFP})$ were then plotted as a function of RS in a histogram (see Figures 2b, 3, and 5).

\section{Simulation of phyllosphere colonization using a} logistic growth model

We adapted a previously developed logistic-growth model for bacteria in a heterogeneous environment (Kinkel et al., 2002). The model simulates a leaf with $S=10^{4}$ sites that vary in their carrying capacity $J$, that is, the maximal number of bacteria that a single site can sustain. Bacteria and their offspring were not allowed to move from one site to another. At the beginning of the simulation, bacteria were distributed over the $S$ sites following a Poisson distribution assuming an initial population of $10^{3}, 10^{4}$ or $10^{5}$ bacteria per leaf. With each iteration $n$, the number of bacteria $N$ in a site doubled, up to a maximum value $N=J$. Heterogeneity in $J$ was introduced by assuming a normal or log-normal distribution that was created using a custom script for NetLogo 4.2.2 (Wilensky, 1999). Other distributions were created with a custom Visual Basic Script for MS Excel (http://azzalini.stat.unipd.it/SN/stephen_gersuk.excel). The trimodal distribution that provided a good fit to the observed experimental data (see Supplementary Information) assumed that $48.5 \%$ of the sites had a $J$ value of 2 , another $48.5 \%$ had a $J$ value of 48 , whereas the remaining $3 \%$ of sites had a $J$ value of 1152 .

\section{Results}

Determining the carrying capacity of bean leaves To determine the bacterial carrying capacity of bean leaves under laboratory conditions, we inoculated four sets of cotyledon leaves with a suspension of E. herbicola strain 299R (Eh299R) CUSPER bacteria to reach four different densities, that is, $4.5 \times 10^{3}$, $6.9 \times 10^{4}, \quad 5.7 \times 10^{5}$ or $1.2 \times 10^{7} \mathrm{CFU}$ per leaf (Figure 1a). Plants were incubated at conditions of
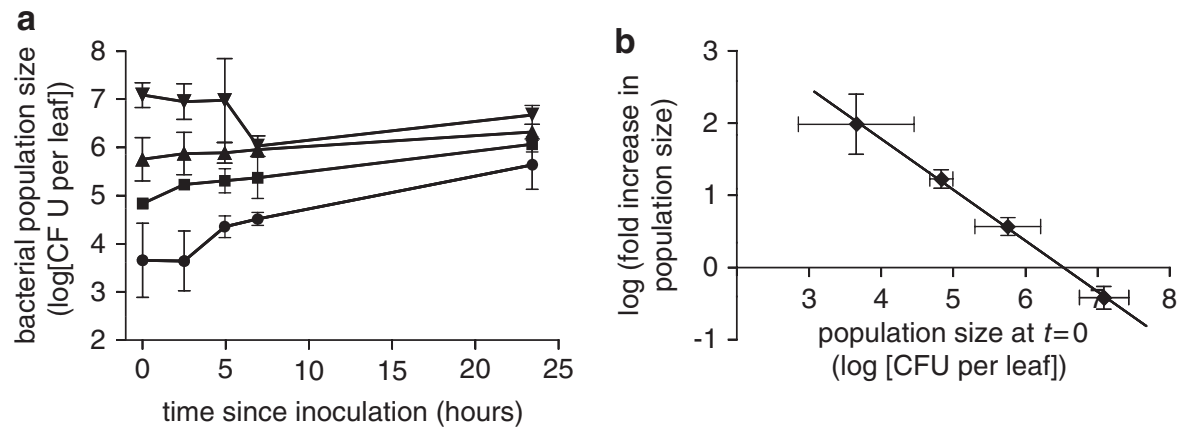

Figure 1 Colonization of bean leaf surfaces by Eh299R CUSPER bacteria at different inoculation densities. (a) Changes in population size were monitored as CFUs, normalized per leaf and plotted as a function of time. Inoculation densities (CFUs per leaf) at $t=0$ :

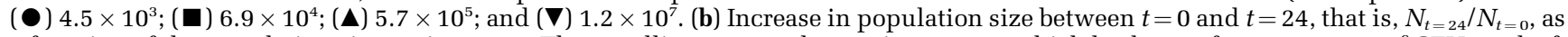
a function of the population size at time $t=0$. The trendline crosses the $x$ axis at 6.535 , which back-transforms to $3.4 \times 10^{6} \mathrm{CFU}$ per leaf. The error bars represent standard deviations from the mean. 
high relative humidity, and we monitored changes in the bacterial population sizes by determining CFU counts per leaf at $3,5,7$ and $24 \mathrm{~h}$ after inoculation (Figure 1a). With the two lowest inoculation densities, population sizes increased during the first $7 \mathrm{~h}$ of the experiment, albeit at different rates. At the two highest inoculation densities, population sizes either remained constant or decreased during this time period. Carrying capacity was estimated from Figure 1b, which shows a plot of the fold increase in population size over $24 \mathrm{~h}$ as a function of the bacterial density at the time of inoculation. The trendline crosses the $x$ axis at $3.4 \times 10^{6} \mathrm{CFU}$ per leaf, which represents the inoculation density where no net growth is expected to occur, that is, the total carrying capacity under these conditions.

\section{Estimating reproductive success (RS) of CUSPER immigrants from GFP content in offspring}

Before introduction into the bean phyllosphere, the CUSPER cells were loaded with stable GFP. This initial amount of GFP gets diluted from cells as they divide (Remus-Emsermann and Leveau, 2010). A hypothetical example is given in Figure 2a to illustrate how GFP content of single CUSPER cell was used to reconstruct the RS of individual immigrants to the leaf surface. In this example, we considered an initial population of $N_{0}=8$ cells. Four of these did not divide, which equaled an RS of 0 for each of those cells. One cell divided once $(\mathrm{RS}=1)$, two divided twice $(\mathrm{RS}=2)$ to produce four cells each and one divided three times $(R S=3)$ for an offspring of eight cells, resulting in a final population at time $t$ of $N_{t}=22$ (Figure 2a). Thus, at time $t$, this yielded four cells with a relative GFP content of 1 (that is, these cells did not divide and GFP was not diluted), two cells with a relative GFP content of 0.5 (each divided once, thus diluting the original GFP into two daughter cells), eight cells with a relative GFP content of 0.25 and eight cells with a relative GFP content of 0.125. For each cell from this population at time $t$, we can calculate two values: (1) GFP/ $\Sigma$ GFP, which is its GFP content divided by the sum of all GFP contents in the same population and (2) ${ }^{2} \log (1 / \mathrm{GFP})$, which is the RS of its progenitor. Cells can then be binned into one of the following RS classes: 0, 1, 2 or 3, and for each bin, the GFP/ $\Sigma$ GFP values of all cells are summed to give $\Sigma(\mathrm{GFP} / \Sigma \mathrm{GFP})$. Figure $2 \mathrm{~b}$ shows $\Sigma(\mathrm{GFP} / \Sigma \mathrm{GFP})$ charted as a function of RS in a bar graph. The inset shows a regular histogram, whereas the larger graph offers a representation in which the width of each bar equals $2^{\text {RS }}$.

It is important to realize that the histogram at any time $t=x$ is constructed solely on the basis of data from time $t=x$ and on the average GFP content at $t=0$. Yet, it tells us many things about the fate of individual cells and the increase in population size since $t=0$. First, the total area covered by all bars in the histogram, that is, $\Sigma\left(\Sigma(\mathrm{GFP} / \Sigma \mathrm{GFP}) \times 2^{\mathrm{RS}}\right)=2.75$, equals the increase in population size between $t=0$ and time $t$, or $N_{t} / N_{0}(22 / 8=2.75)$. This value can a

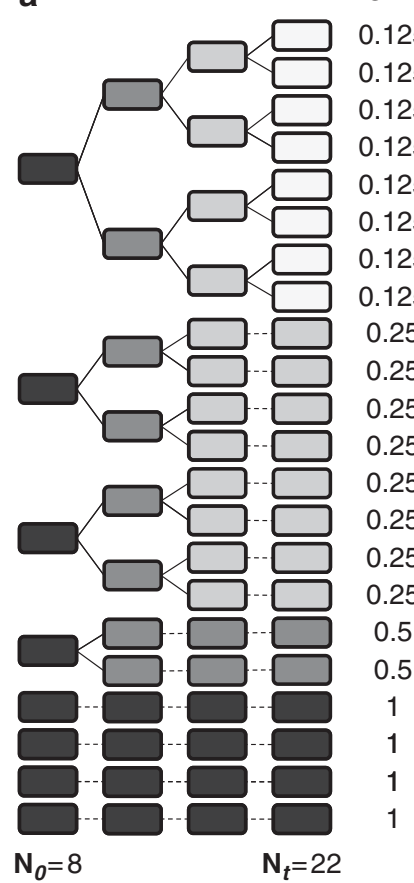

$\begin{array}{ccc}\text { GFP } & \text { GFP/ } \Sigma \text { GFP } & { }^{2} \log (1 / G F P) \\ 0.125 & 0.015625 & 3 \\ 0.125 & 0.015625 & 3 \\ 0.125 & 0.015625 & 3 \\ 0.125 & 0.015625 & 3 \\ 0.125 & 0.015625 & 3 \\ 0.125 & 0.015625 & 3 \\ 0.125 & 0.015625 & 3 \\ 0.125 & 0.015625 & 3 \\ 0.25 & 0.03125 & 2 \\ 0.25 & 0.03125 & 2 \\ 0.25 & 0.03125 & 2 \\ 0.25 & 0.03125 & 2 \\ 0.25 & 0.03125 & 2 \\ 0.25 & 0.03125 & 2 \\ 0.25 & 0.03125 & 2 \\ 0.25 & 0.03125 & 2 \\ 0.5 & 0.0625 & 1 \\ 0.5 & 0.0625 & 1 \\ 1 & 0.125 & 0 \\ 1 & 0.125 & 0 \\ 1 & 0.125 & 0 \\ 1 & 0.125 & 0 \\ & & \end{array}$

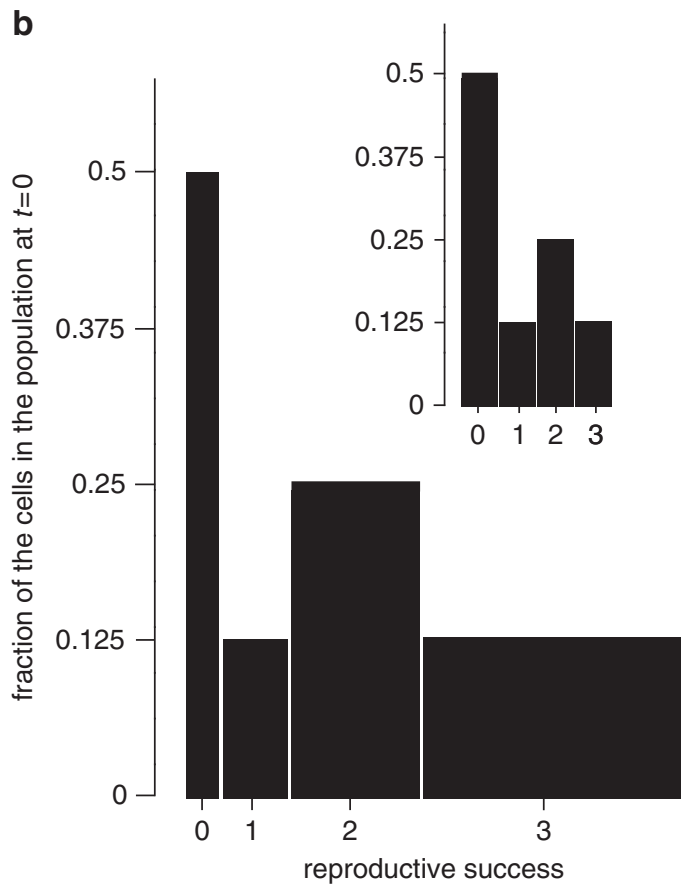

Figure 2 Illustrative example of how GFP content in CUSPER offspring at time $t$ can be retropolated to reveal the RS of bacterial cells at time $t=0$. Details are provided in the text. 
also be calculated directly from $N_{t} / \Sigma \mathrm{GFP}$, that is, the number of cells at time $t$ divided by the sum of all GFP contents. Second, the graph reveals how successful different fractions of the original population were in producing offspring. For example, it shows that half (that is, $\Sigma(\mathrm{GFP} / \Sigma \mathrm{GFP})=0.5)$ of the cells in the population at $t=0$ did not divide $(\mathrm{RS}=0)$ and that a quarter of the cells $(\Sigma(\mathrm{GFP} /$ $\Sigma \mathrm{GFP})=0.25$ ) at $t=0$ divided two times to produce four cells at time $t(\mathrm{RS}=2)$. Third, the area of any single bar in the graph divided by the total area covered by all bars in the histogram gives the proportional contribution of cells from the corresponding RS bin to the population size at time $t$. For example, the area of the third bar $(\mathrm{RS}=2)$ is $\Sigma(\mathrm{GFP} /$ $\Sigma$ GFP) $\times 2^{\mathrm{RS}}=0.25 \times 2^{2}=1$, which, divided by 2.75 (=total area, see above), equals 0.36 , meaning that $25 \%$ of the cells in the original population divided twice to produce enough offspring to account for $36 \%$ of the population at time $t$.

\section{Single-cell experiences of leaf colonization after} inoculation at different densities

Using the analysis illustrated in Figure 2, we retropolated GFP content of leaf-recovered bacteria to the RS of individual bacteria in the $t=0$ population. Specifically, we analyzed bacterial cells retrieved at the $t=24$ time point from eight leaves (four inoculation densities, two leaf washings each) from the experiment for which population data are shown in Figure 1. The results are summarized in Figure 3, which shows eight histograms $(\mathrm{a}-\mathrm{h})$, that is, two leaves from different plants for each density. These graphs indicate great variation in the fate of individual bacteria after they were introduced onto the leaf surface at different starting densities.

On the two leaves that received the lowest inoculation density (Figures $3 \mathrm{a}$ and $\mathrm{b}$ ), $41 \%$ and $70 \%$, respectively, of the initial colonizers divided once after arrival on the bean leaf surface. We interpret this to mean that a substantial portion of the immigrant population landed in a spot that did not support much growth. On the other hand, 59\% and $21 \%$, respectively, of the cells at $t=0$ was very successful, with an RS value of 5 or greater, that is, they produced $\geqslant 32$ offspring. These very successful immigrants reproduced to become $98 \%$ and $80 \%$, respectively, of the final population size, compared with only $2 \%$ and $13 \%$, respectively, for the cells that underwent only one division upon arrival.

With increasing inoculation density, the relative fraction of very successful cells $(\mathrm{RS} \leqslant 5)$ diminished (Figure 3), down to $2.4 \%$ and $0.7 \%$, respectively, on the two leaves that were sprayed with the highest inoculation density (Figures $3 \mathrm{~g}$ and $\mathrm{h}$ ). At this density, $91 \%$ and $93 \%$, respectively, of the bacterial immigrants each produced four offspring at most, with a majority not dividing at all or only once. Despite their low initial abundance of $2.4 \%$ and $0.7 \%$, the very successful cells contributed a considerable fraction, $31 \%$ and $11 \%$, respectively, of the final population size.

It is evident from Figure 3 that with increasing inoculation density, the sum of the area underneath all of the bars (representing $N_{24} / N_{0}$, as explained in Figure 2) decreases. This decline in fold increase in population size is consistent with our findings in Figure 1b, and can be explained by assuming that at higher inoculation densities, similar amounts of resources need to be shared by more bacteria, resulting in fewer offspring per cell. The notion that there were still few very successful immigrants at the highest inoculation density suggests that a small portion of the leaf surface $(2.4 \%$ and $0.7 \%$ in this case) consisted of sites that allow considerably more reproduction than most other sites. Another trend that is apparent in Figure 3 is the increase in resemblance of the two histograms from each inoculation density, as inoculation density increases. This suggests that the course and outcome of leaf colonization become more predictable at higher inoculation densities.

For each of the eight histograms shown in Figure 3, we plotted in Figure 4 the sum of the area underneath all of the bars as a function of the $N_{24} / N_{0}$ fold increase that was obtained from CFU-based data. The fact that data points did not fall on a line with slope 1 through the origin indicates that GFP- and CFU-based results do not fully agree with each other. At the lowest inoculation density, this discrepancy can be explained by the known limitation of CUSPER (Remus-Emsermann and Leveau, 2010) that after five cell divisions GFP gets diluted to the point where we can no longer reliably detect and quantify it. For the two leaves inoculated at this density, we calculated that in order to get the GFPbased estimates for increase in population size up to the one derived from CFU data, one would have to assume average RS values of $7.4 \%$ and $9.0 \%$ respectively, for cells that were binned in the RS $>5$ category in Figure 3. This corresponds to 169 and 512 offspring, respectively, for each of these cells. At the highest inoculation density, the discrepancy between GFP- and CFU-based results may be explained by assuming that a proportion of the cells on the leaf were not able to form a colony on the plate. For the two leaves that were inoculated at this density, we calculated that in order to get the CFU-based estimates for increase in population size to match those derived from GFP data, one would have to assume that $90 \%$ and $87 \%$, respectively, of the cells lost their ability to form a colony between $t=0$ and $t=24$. Another hypothetical explanation for the discrepancy between CFU- and GFP-based data is that aggregates that were washed from the leaf surface did not break up into single cells during the wash procedure, so that one CFU might represent more than one cell. However, we can exclude this possibility, based on the fact that such aggregates were never observed under the microscope. 

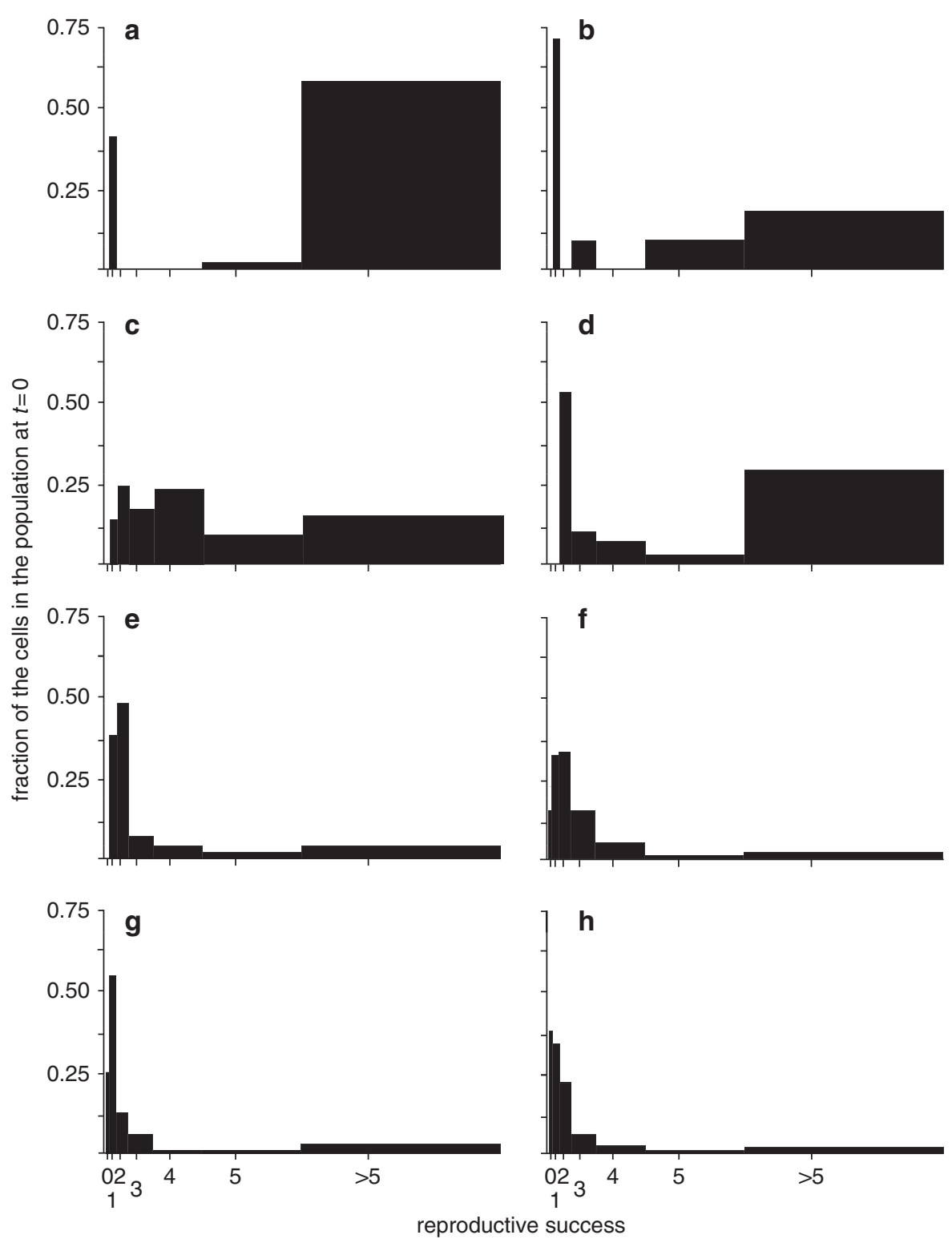

Figure 3 Variation in RS of CUSPER immigrants to the bean leaf surface, as inferred from GFP content in bacteria that were retrieved from leaves after $24 \mathrm{~h}$ of incubation. Cells were retrieved from the same experimental setup that was used to construct Figure 1. For each of the four inoculation densities, cells from two independent leaf samples were analyzed: (a and b) $4.5 \times 10^{3} \mathrm{CFU}$ per leaf; (c and d) $6.9 \times 10^{4} \mathrm{CFU}$ per leaf; (e and f) $5.7 \times 10^{5} \mathrm{CFU}$ per leaf; and ( $\mathrm{g}$ and $\mathbf{h}$ ) $1.2 \times 10^{7} \mathrm{CFU}$ per leaf. Histograms were fashioned in the same way as was done for the fictional data in Figure 2.

Modeling single-cell experiences in the phyllosphere We used our experimental data to test a modified version of a previously formulated logistic growth model for bacterial colonization of a heterogeneous leaf environment (Kinkel et al., 2002). Our model consisted of a virtual leaf that contains $10^{4}$ independent sites. Leaves were inoculated with $10^{3}, 10^{4}$ or $10^{5}$ virtual bacteria following a Poisson distribution. For the lowest inoculation density, this means that $\sim 90 \%$ of all sites were devoid of bacteria, whereas at the highest inoculation density each site contained 10 cells on average. In each site, bacteria were allowed to reproduce up to the site's local carrying capacity $J$. We tested different distributions of $J$ and recorded the RS of individual bacteria. We found that of all the tested scenarios, a trimodal (low, medium and high) distribution of $J$ was the one that best matched the general patterns that we observed for the experimental data (Figure 5), that is, a co-occurrence of very successful and nearly unsuccessful immigrants at low inoculation densities and the existence of a small portion of very successful colonizers among a majority of less successful immigrants at high inoculation densities. The best fitting distribution assumed roughly similar numbers of sites with low or medium local 


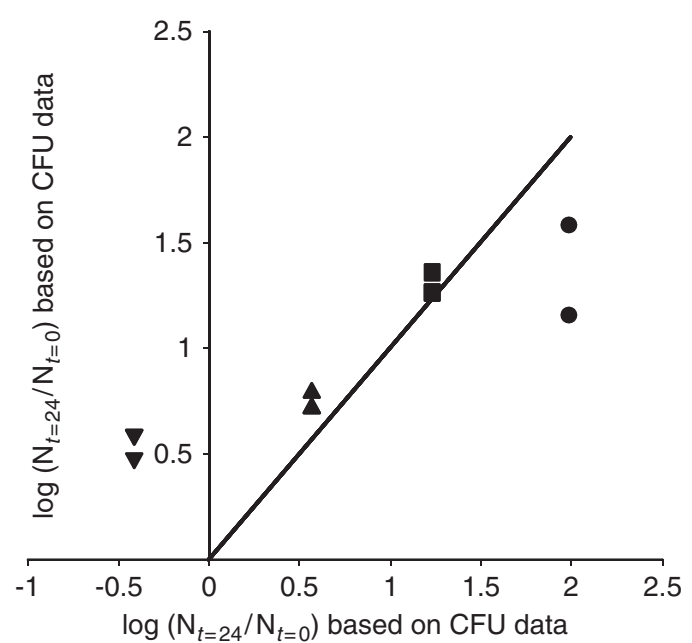

Figure 4 Comparison of estimates for $N_{t=24} / N_{t=0}$, that is, fold increase in bacterial population sizes over $24 \mathrm{~h}$, based on CFU data and GFP data. For the $x$ values of each data point, we used the $y$ axis values shown in Figure 1b. For the $y$ values of each data point, we used the total area under all bars for each corresponding histogram shown in Figure 3. The symbols mark the inoculation density (CFU per leaf) at $t=0$ : (O) $4.5 \times 10^{3}$; (目 $6.9 \times 10^{4}$; (A) $5.7 \times 10^{5}$; and $(\boldsymbol{\nabla}) 1.2 \times 10^{7}$, and correspond to the symbols used in Figure 1. If the CFU- and GFP-based estimates for one particular inoculation density were identical, the corresponding data point would fall on the line with slope 1 . The deviations from this line at low and high inoculation densities are discussed in the text.

carrying capacity, whereas the remaining very small portion of sites was characterized by high local carrying capacity. In the model, a high inoculation density meant that medium- $J$ sites received immigrants in numbers that for the most part approximated or exceeded $J$, such that these cells did not differ much in RS from cells that landed in low- $J$ sites. In contrast, the few high- $J$ sites were occupied by cells in numbers well below $J$, allowing for a substantial number of offspring per cell. At low inoculation density, the low frequency of high- $J$ sites prevented most, if not all, cells from landing in one of these sites, but as medium- $J$ sites received cells in numbers well below $J$, there was still an opportunity for these cells to proliferate much more than cells that landed in low- $J$ sites, where no or few doublings occurred.

\section{Discussion}

We have determined the bacterial carrying capacity of bean leaves under controlled conditions in the laboratory. By bioreporter analysis, we were able to demonstrate that under these conditions, realized carrying capacity at different inoculation densities is a function of variation in the local carrying capacity of different sites on the leaf surface. Our findings are consistent with a model of phyllosphere colonization that features different fates for bacterial immigrants, depending on where and with how many others they land on the leaf surface. Our study offers a quantitative estimate for these fates, which is a significant step toward a more predictive understanding of bacterial colonization of leaf surfaces.

It is evident from our study that individual- and population-based data sets each offer an incomplete understanding of how bacteria colonize a leaf. CUSPER-based measurements have the limitation that information is lost beyond a RS of 5 (RemusEmsermann and Leveau, 2010), so that the contribution of some immigrants to the population will be underestimated, whereas CFU-based measurements provide only the average contribution of cells to the population, thus ignoring the variability that clearly exists among immigrants. In combination, however, GFP- and CFU-based measurements offered significant novel insights. For example, the substantial number of cells that divide at most once upon arrival to the leaf surface at low inoculation density, and the corresponding high increase in population size as derived from CFU counts, suggests that the RS of cells that actually did divide was underestimated and that some cells could produce offspring in numbers that exceed 500 cells. Bacterial aggregates of this size and much larger have been found on bean leaves under appropriate conditions (Monier and Lindow, 2004), although it was not established whether they were derived from single cells. On the other hand, to explain high RS values for individual bacterial immigrants at high inoculation densities in the context of a constant or decreasing CFU count under the same conditions, one needs to assume that a substantial number of cells lose their ability to form a colony on agar plates. Indeed, such nonculturability has been demonstrated experimentally for $P$. syringae, another model colonizer of the phyllosphere, even under favorable conditions of relative high humidity (Wilson and Lindow, 1992). There is good evidence in the literature (for example, Unge et al., 1999; Lowder et al., 2000) that the GFP variant that we used in our study is extremely stable and continues to fluoresce in cells that enter this state of nonculturability.

In our current model of bacterial colonization of the phyllosphere, each leaf colonization event can be thought of as a bottleneck occurrence, where some percentage of the incoming population is prevented from reproducing, or even surviving, whereas other immigrants in that same population are numerically enriched because they settle in sites conducive to growth. On the basis of our derived estimates on the quantity and quality of sites, landing in a high- $J$ site is unlikely for any individual bacterium, and at low inoculation densities, chance will determine whether such sites will be filled or not. Thus, the outcome of an inoculation event would be less predictable for low inoculation densities than for high ones. 

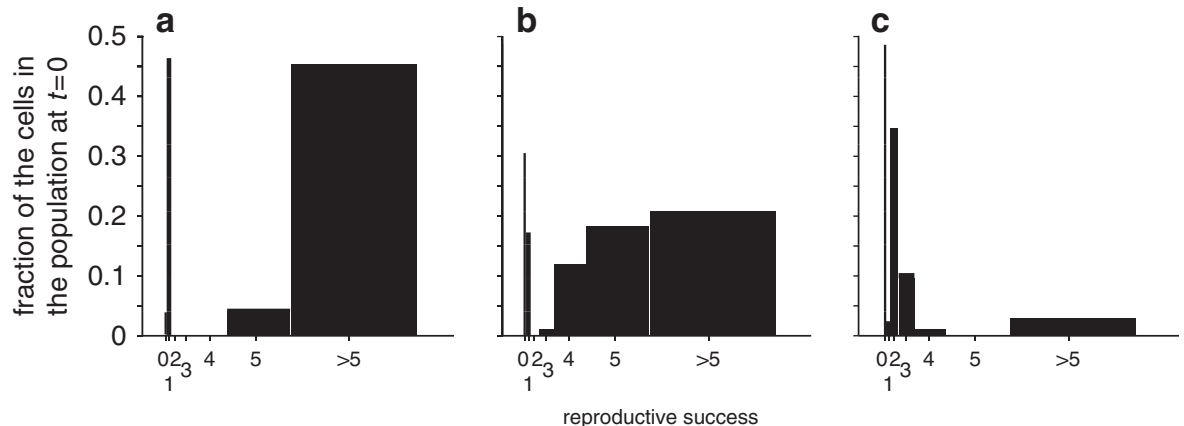

Figure 5 Variation in RS of virtual bacterial immigrants to a simulated leaf environment. The model is described in the text. It assumes a leaf with $10^{4}$ discrete sites that vary in their carrying capacity $J$. This leaf was inoculated with $10^{3}(\mathbf{a}), 10^{4}(\mathbf{b})$ or $10^{5}(\mathbf{c})$ bacteria per leaf. For this simulation, we assumed that $J=2$ for $48.5 \%$ of the sites, $J=48$ for another $48.5 \%$ of the sites and $J=1152$ for the remaining $3 \%$ of the sites.

Ecological theory holds that if a nutrient resource is limited and the number of organisms competing for this resource is high, the average success of the population goes down (Galliard et al., 2005; Rankin and Kokko, 2006). This is precisely what we observed in our experiments and simulations: high inoculation densities forced more bacterial individuals to share a site and resulted in overall reduced RS. This seems to support the notion that local carrying capacity on leaves is determined by local nutrient availability. Being perfectly isogenic, CUSPER cells can be considered each other's ultimate competitors, with a niche overlap index of 1 (Wilson and Lindow, 1994a,b). Thus, unless a site offered very high levels of nutrients, bacterial immigrants would be relatively unable to reproduce, representing a convincing case of the 'tragedy of the commons' (Hardin, 1968). However, a one-time immigration event of up to $10^{7}$ bacteria per leaf is not a likely event in nature (Lindemann and Upper, 1985). Instead, immigration is a gradual process, with cells arriving to the leaf from the surrounding air at different times and typically at low rates (Upper and Hirano, 2002). Another aspect of our experimental setup that can be considered artefactual is that all immigrants were of clonal descent. In reality, the leaf surface is colonized by very diverse bacteria and other microorganisms with different niche overlap indexes, which allows for various degrees of coexistence (Knief et al., 2010a). However, it is important to note that the ability to set up such artificial colonization events allowed us to extract data on the quality of the leaf surface as a habitable environment. This high level of experimental amenability is quite unique to microbial ecology and is one of the advantages microbial ecologists have over 'macro'-bial ecologists (Fierer, 2007). Future applications of CUSPER will address more realistic scenarios by assessing variation in RS of CUSPER cells in competition with other bacterial species and after immigration onto precolonized leaves.

Our observation that a small fraction (0.7-2.4\%) of the cells was very successful in reproducing even at the highest inoculation density suggests the existence of sites on the leaf surface that offer high levels of nutrients, allowing these cells to contribute disproportionally $(11-31 \%)$ to the final population size. Interestingly, this finding agrees with reports showing that relatively rare features on the leaf surface tend to harbor large numbers of bacteria on colonized leaves. Specifically, it is worth noting that only $0.6 \%$ of the bean leaf surface is covered by hooked and glandular trichomes (Monier and Lindow, 2005a,b), yet $44.1 \%$ of Eh299R bacteria in aggregates were found to be associated with these structures after incubation under moist conditions for 5 days (Monier and Lindow, 2005a, b). In at least one study (Yadav et al., 2005), the population size of epiphytic bacteria was positively correlated with the densities of glandular and nonglandular trichomes. This link between bacterial fate, population size and leaf topography calls for further investigation into the predictive value of trichome density for bacterial colonization of leaf surfaces.

As was pointed out before (Remus-Emsermann and Leveau, 2010), application of the CUSPER concept is not limited to leaf surfaces, but can be extended widely to many other bacterial habitats. Several bioreporter-based studies have revealed variation in the bacterial experience of such habitats (Moller et al., 1998; Jaspers et al., 2001; Herron et al., 2010), and all point to microscale heterogeneity in terms of the physical, chemical and biological conditions that an individual bacterium can be exposed to in a given environment. The CUSPER approach offers an insight in bacterial fate, which does not follow from knowing exactly what these conditions were; instead, it settles for knowing that these conditions were (or were not) permissive to the production of offspring. This interpretation of environmental heterogeneity matches quite well with the concept of local carrying capacity, where the microbial landscape, be it natural or man-made (for example, Keymer et al., 2006; Battin et al., 2007) features local variation in the ability of patches to sustain bacterial growth. 


\section{Acknowledgements}

The study was funded by the Netherlands Organisation of Scientific Research (NWO) in the form of a personal VIDI grant to JHJL. This is NIOO-KNAW publication number 5156.

\section{References}

Battin TJ, Sloan WT, Kjelleberg S, Daims H, Head IM, Curtis TP et al. (2007). Microbial landscapes: new paths to biofilm research. Nat Rev Microbiol 5: 76-81.

Elad Y, Kirshner B. (1993). Survival in the phylloplane of an introduced biocontrol agent Trichoderma harzianum and populations of the plant pathogen Botrytis cinerea; as modified by abiotic conditions. Phytoparasitica 21: 303-313.

Fierer N. (2007). Finding a place for microorganisms in the field of ecology. Ecology 88: 1336-1337.

Hardin G. (1968). The tragedy of the commons. Science 162: 1243-1248.

Herron PM, Gage DJ, Cardon ZG. (2010). Micro-scale water potential gradients visualized in soil around plant root tips using microbiosensors. Plant Cell Environ 33: 199-210.

Jacobs JL, Carroll TL, Sundin GW. (2005). The role of pigmentation, ultraviolet radiation tolerance, and leaf colonization strategies in the epiphytic survival of phyllosphere bacteria. Microb Ecol 49: 104-113.

Jacques MA, Kinkel LL, Morris CE. (1995). Population sizes, immigration, and growth of epiphytic bacteria on leaves of different ages and positions of field-grown endive (Cichorium endivia var. latifolia). Appl Environ Microbiol 61: 899-906.

Jaspers MCM, Meier C, Zehnder AJ, Harms H, van der Meer JR. (2001). Measuring mass transfer processes of octane with the help of an alkS-alkB::gfp-tagged Escherichia coli. Environ Microbiol 3: 512-524.

Keymer JE, Galajda P, Muldoon C, Park S, Austin RH. (2006). Bacterial metapopulations in nanofabricated landscapes. Proc Natl Acad Sci USA 103: 17290-17295.

Kinkel LL, Newton M, Leonard KJ. (2002). Resource aggregation in the phyllosphere: implications for microbial dynamics across spatial scales. In: Lindow S, Hecht-Poinar E, Elliott V (eds). Phyllosphere Microbiology. APS Press: Saint Paul, MN, USA, pp 317-340.

Kinkel LL, Wilson M, Lindow SE. (2000). Plant species and plant incubation conditions influence variability in epiphytic bacterial population size. Microb Ecol 39: 1-11.

Knief C, Frances L, Vorholt JA. (2010a). Competitiveness of diverse Methylobacterium strains in the phyllosphere of Arabidopsis thaliana and identification of representative models, including $M$. extorquens PA1. Microb Ecol 60: 440-452.

Knief C, Ramette A, Frances L, Alonso-Blanco C, Vorholt JA. (2010b). Site and plant species are important determinants of the Methylobacterium community composition in the plant phyllosphere. ISME $J$ 4: 719-728.

Leben C. (1965). Influence of humidity on migration of bacteria on cucumber seedlings. Can J Microbiol 11: 671-676.
Le Galliard JF, Fitze PS, Ferrière R, Clobert J. (2005). Sex ratio bias, male aggression, and population collapse in lizards. Proc Natl Acad Sci USA 102: 18231-18236.

Leveau JHJ. (2006). Microbial Communities in the Phyllosphere. Biology of the Plant Cuticle. Riederer M and Müller C, Blackwell Publishing Ltd: Oxford, UK, pp 334-367.

Leveau JHJ, Lindow SE. (2001a). Appetite of an epiphyte: quantitative monitoring of bacterial sugar consumption in the phyllosphere. Proc Natl Acad Sci USA 98: 3446-3453.

Leveau JHJ, Lindow SE. (2001b). Predictive and interpretive simulation of green fluorescent protein expression in reporter bacteria. J Bacteriol 183: 6752-6762.

Lindemann J, Upper CD. (1985). Aerial dispersal of epiphytic bacteria over bean plants. Appl Environ Microbiol 50: 1229-1232.

Lindow SE, Brandl MT. (2003). Microbiology of the phyllosphere. Appl Environ Microbiol 69: 1875-1883.

Lowder M, Unge A, Maraha N, Jansson JK, Swiggett J, Oliver JD. (2000). Effect of starvation and the viable-but-nonculturable state on green fluorescent protein (GFP) fluorescence in GFP-tagged Pseudomonas fluorescens A506. Appl Environ Microbiol 66: $3160-3165$.

McArthur JV. (2006). Microbial Ecology: An Evolutionary Approach. Academic Press: Oxford, UK.

Mercier J, Lindow SE. (2000). Role of leaf surface sugars in colonization of plants by bacterial epiphytes. Appl Environ Microbiol 66: 369-374.

Moller S, Sternberg C, Andersen JB, Christensen BB, Ramos JL, Givskov $M$ et al. (1998). In situ gene expression in mixed-culture biofilms: evidence of metabolic interactions between community members. Appl Environ Microbiol 64: 721-732.

Monier JM, Lindow SE. (2004). Frequency, size, and localization of bacterial aggregates on bean leaf surfaces. Appl Environ Microbiol 70: 346-355.

Monier JM, Lindow SE. (2005a). Aggregates of resident bacteria facilitate survival of immigrant bacteria on leaf surfaces. Microb Ecol 49: 343-352.

Monier JM, Lindow SE. (2005b). Spatial organization of dual-species bacterial aggregates on leaf surfaces. Appl Environ Microbiol 71: 5484-5493.

Nix S, Burpee LL, Buck JW. (2009). Responses of 2 epiphytic yeasts to foliar infection by Rhizoctonia solani or mechanical wounding on the phylloplane of tall fescue. Can J Microbiol 55: 1160-1165.

Rankin DJ, Kokko H. (2006). Sex, death and tragedy. Trend Ecol Evol 21: 225-226.

Remus-Emsermann MNP, Leveau JHJ. (2010). Linking environmental heterogeneity and reproductive success at single-cell resolution. Isme J 4: 215-222.

Tukey HB. Jr. (1966). Leaching of metabolites from above-ground plant parts and its implications. Bulletin Torrey Bot Club 93: 385-401.

Unge A, Tombolini R, Molbak L, Jansson JK. (1999). Simultaneous monitoring of cell number and metabolic activity of specific bacterial populations with a dual gfp-lux $A B$ marker system. Appl Environ Microbiol 65: 813-821.

Upper C, Hirano S. (2002). Revisiting the roles of immigration and growth in the development of populations of Pseudomonas syringae in the 
phyllosphere. In: Lindow S, Hecht-Poinar E, Elliott V (eds). Phyllosphere Microbiology. APS Press: Saint Paul, MN, USA, pp 69-79.

Van Der Wal A, Leveau JHJ. (2010). Modelling sugar diffusion across plant leaf cuticles: the effect of free water on substrate availability to phyllosphere bacteria. Environ Microbiol 13: 792-797.

Wilensky U. (1999). NetLogo. Center for Connected Learning and Computer-Based Modeling, Northwestern University: Evanston, IL.

Wilson M, Lindow S. (1992). Relationship of total viable and culturable cells in epiphytic populations of Pseudomonas syringae. Appl Environ Microbiol 58: 3908-3913.

Wilson M, Lindow S. (1995). Enhanced epiphytic coexistence of near-isogenic salicylate-catabolizing and non-salicylate-catabolizing Pseudomonas putida strains after exogenous salicylate application. Appl Environ Microbiol 61: 1073-1076.

Wilson M, Lindow SE. (1994a). Ecological similarity and coexistence of epiphytic ice-nucleating (Ice+) Pseudomonas syringae strains and a non-ice-nucleating (Ice-) biological control agent. Appl Environ Microbiol 60: 3128.
Wilson M, Lindow SE. (1994b). Inoculum density-dependent mortality and colonization of the phyllosphere by Pseudomonas syringae. Appl Environ Microbiol 60: 2232-2237.

Wilson M, Savka MA et al. (1995). Altered epiphytic colonization of mannityl opine-producing transgenic tobacco plants by a mannityl opine-catabolizing strain of Pseudomonas syringae. Appl Environ Microbiol 61: 2151-2158.

Woody ST, Ives AR, Nordheim EV, Andrews JH. (2007). Dispersal, density dependence, and population dynamics of a fungal microbe on leaf surfaces. Ecology 88: $1513-1524$.

Yadav R, Karamanoli K, Vokou D. (2005). Bacterial colonization of the phyllosphere of Mediterranean perennial species as influenced by leaf structural and chemical features. Microb Ecol 50: 185-196.

(c) This work is licensed under the Creative Commons Attribution-NonCommercial-No Derivative Works 3.0 Unported License. To view a copy of this license, visit http://creativecommons. org/licenses/by-nc-nd/3.0/

Supplementary Information accompanies the paper on The ISME Journal website (http://www.nature.com/ismej) 